IUHET-503

\title{
Bounds on Spin-Dependent Lorentz Violation From Inverse Compton Observations
}

\author{
Brett Altschul] \\ Department of Physics \\ Indiana University \\ Bloomington, IN 47405 USA
}

\begin{abstract}
Some of the best bounds on possible Lorentz violation in the electron sector come from observations of high-energy astrophysical phenomena. Using measurements of $\mathrm{TeV}$ inverse Compton radiation from a number of sources, we place the first bounds - at the $10^{-15}$ level — on seven of the electron $d$ coefficients.
\end{abstract}

\footnotetext{
${ }^{1}$ baltschu@indiana. edu
} 
In the last ten years, there has been growing interest in the possibility that special relativity may not be exactly correct. Lorentz invariance might be only approximately valid, with there existing small deviations from rotation and boost invariance. A variety of experimental tests have been performed, constraining many types of Lorentz violations to be small. However, many other quite reasonable forms of Lorentz violation are poorly, if at all, constrained.

In quantum field theory language, Lorentz violation is described by the standard model extension (SME) [1, 2]. Lorentz-violating corrections to gravity can also be incorporated [3]. The minimal SME, which contains gauge invariant, renormalizable parameters, provides a convenient parameterization of possible Lorentz violations, in terms of various background tensor coefficients. There are separate sets of coefficients for each species of particle in the theory.

In this paper, we are specifically interested in electron Lorentz violation. Bounds on the Lorentz-violating coefficients for electrons come from clock comparison experiments [4, 5], experiments with spin-polarized matter [6, 7], Michelson-Morley experiments with cryogenic resonators [8, 10, 9, 11], and Doppler effect measurements [12, 13]. Finally, some of the best bounds come from high-energy astrophysics [14, 15, 16, 17, 18. A number of astrophysical processes have been used to set these bounds. We shall show that observations of one of these processes, inverse Compton (IC) scattering, can be used to place tight bounds on seven SME parameters that have not been bounded before.

The minimal SME Lagrange density for the electron sector is

$$
\mathcal{L}=\bar{\psi}\left(i \Gamma^{\mu} \partial_{\mu}-M\right) \psi
$$

where

$$
M=m+\not \alpha-\not \gamma \gamma_{5}+\frac{1}{2} H^{\mu \nu} \sigma_{\mu \nu}+i m_{5} \gamma_{5}
$$

and

$$
\Gamma^{\mu}=\gamma^{\mu}+c^{\nu \mu} \gamma_{\nu}-d^{\nu \mu} \gamma_{\nu} \gamma_{5}+e^{\mu}+i f^{\mu} \gamma_{5}+\frac{1}{2} g^{\lambda \nu \mu} \sigma_{\lambda \nu}
$$

However, some of the coefficients, such as $f$ and $a$, are unphysical [19]. At high energies, the Lorentz-violating effects coming from $\Gamma^{\mu}$ are more important than those coming from $M$, simply because the $\Gamma^{\mu}$ is multiplied by a factor of the momentum. The coefficients contained in $M$ do not affect the maximum achievable velocity (MAV) of the particles, but the ones included in $\Gamma^{\mu}$ do.

In order to canonically quantize the electron field, we must have $\Gamma^{0}=\gamma^{0}$ in the frame where the quantization is to be performed. Otherwise, there are nonstandard time derivatives that make defining a Hamiltonian impossible. If all Lorentz violations are small, it is possible to perform a rotation in spinor space so that in the new basis, $\Gamma^{0}$ does not contain any unconventional terms. Then, in the frame in which the theory is quantized, we must have $c^{\nu 0}=d^{\nu 0}=e^{0}=g^{\lambda \nu 0}=0$. We shall adopt this convention 
here, choosing as the quantization frame the rest frame of the sun and using sun-centered celestial equatorial coordinates $(X, Y, Z, T)[20]$.

In the full SME, the $e$ and $g$ terms are actually forbidden if we demand renormalizability. These terms mix left- and right-handed leptons and hence break $S U(2)_{L}$ gauge invariance. This gauge invariance must be present prior to spontaneous symmetry breaking if the theory is to be renormalizable; so $e$ and $g$ could only arise as part of the spontaneous breaking. However, the operators parameterized by $e$ and $g$ are of dimension four, so they could only appear as vacuum expectation values of higher-dimension, nonrenormalizable operators. Even if we do not insist on renormalizability, we would expect nonrenormalizable terms like $e$ and $g$ to be suppressed relative to the renormalizable ones.

The renormalizable operators contained in $\Gamma^{\mu}$ are described by $c$ and $d$. The $c$ terms and $d$ terms are similar in form, although the former are obviously simpler. There are already some fairly good bounds on the $c$ terms in the electron sector, most of them coming from astrophysical data; however, the $d$ terms have been less explored. At nonrelativistic energies, the effects of $d$ must enter in specific combinations of $d$ and $H$, and all existing bounds on $d$ are actually bounds on these particular combinations. However, using relativistic tests, bounds on $d$ may be obtained separately from $H$.

Our focus in this paper will be on the $d$ terms and how they affect the MAV of electrons. The effects of $c$ have previously been considered [17, 18]; with a $c$ term only, the maximum electron velocity in a direction $\hat{e}$ is

$$
\left(v_{j} \hat{e}_{j}\right)_{\max }=1-c_{j k} \hat{e}_{j} \hat{e}_{k}-c_{0 j} \hat{e}_{j}
$$

If this is less than one in a given direction, there will be a maximum Lorentz factor $\gamma=\left(1-\vec{v}^{2}\right)^{-1 / 2}$ for particles moving in that direction, which would have observable effects on the synchrotron spectra of high-energy sources. On the other hand, if $\left(v_{j} \hat{e}_{j}\right)_{\max }$ were greater than one, there would exist a finite maximum energy for particles with speeds less than one. More energetic particles, moving faster than the speed of light, would lose energy quickly through vacuum Cerenkov radiation. Lower limits on electrons' maximum subluminal energies can be inferred from sources' IC spectra. Combining the synchrotron and IC information for a single source, we can obtain a two-sided bound on a particular linear combination of the $c^{\nu \mu}$ coefficients.

By observing the radiation from electrons of energy $E$, we could place bounds on $c$ that are $\mathcal{O}\left(m^{2} / E^{2}\right)$. This energy scale at which phenomena such as vacuum Cerenkov radiation and single-photon pair creation would appear is $m / \sqrt{|c|}[21]$, and so the absence of these effects up to a scale $E$ indicates $|c|$ must be smaller than the indicated $\mathcal{O}\left(\mathrm{m}^{2} / E^{2}\right)$. The forms of Lorentz violation parameterized by $c$ are especially simple; the effects of $c$ are independent of particle spin, and the $c$ term in the Lagrangian is even under both $C$ and $P T$.

The situation with a $d$ instead of a $c$ is trickier. The $d$ term in $\Gamma^{\mu}$ differs only from the $c$ term by the presence of a $\gamma_{5}$, but it is odd under $C$ and PT. At ultrarelativistic energies 
where the mass can be neglected, $\gamma_{5}$ is the helicity operator and can be diagonalized simultaneously with the Hamiltonian. So at these high energies, $d$ resembles a $c$ whose sign depends on the helicity. Therefore, the sign of contribution of $d$ to the MAV is helicity dependent, and hence there can never be a MAV less than one. (One helicity will have a MAV less than one, but the other will not. In real sources, the spins are not polarized, and so the physically relevant MAV must be a maximum over all spin states.) However, it turns out that IC data can actually give two-sided bounds on $d$ by constraining the possibility that the MAV may be greater than one, even though the same kinds of data only give one-sided bounds on $c$.

With a $d$ term only, we would expect that the MAV in a given direction would become

$$
\left(v_{j} \hat{e}_{j}\right)_{\max }=1+s d_{j k} \hat{e}_{j} \hat{e}_{k}+s d_{0 j} \hat{e}_{j},
$$

where $s$ is now the helicity, just based on the similar way that $c$ and $d$ enter into $\mathcal{L}$. However, $\gamma_{5}$ is strictly only the helicity operator when the electron mass vanishes. The mass will enter unavoidably into our bounds, and this raises the question of whether simply making the replacement $\gamma_{5} \rightarrow s$ is really valid. This is most easily answered by looking at the explicit energy-momentum relation and group velocity in the presence of both $d$ and $m$; this has been done in [22]. The dispersion relation is simplest if only $d_{0 j}$ is nonzero, when we have

$$
E^{2}=m^{2}+\left(|\vec{\pi}|+s d_{0 j} \pi_{j}\right)^{2}
$$

and

$$
\left(v_{g}\right)_{k}=\frac{|\vec{\pi}|+s d_{0 j} \pi_{j}}{E}\left(\hat{\pi}_{k}+s d_{0 k}\right) .
$$

The maximum speed for a given helicity $s$ is, to leading order in $d, 1+s d_{0 j} \hat{\pi}_{j}$, and the velocity is superluminal if

$$
s d_{0 j} \hat{\pi}_{j}<-\frac{1}{2(E / m)^{2}} .
$$

The energy-momentum relation with $d_{j k}$ is substantially more cumbersome, but the pattern is the same. The net result is that $v>1$ whenever

$$
s d_{0 j} \hat{\pi}_{j}+s d_{j k} \hat{\pi}_{j} \hat{\pi}_{k}<-\frac{1}{2(E / m)^{2}}
$$

It is obvious that by choosing one or the other sign for $s$, we can ensure that the inequality (9) does not hold and so the electron is moving more slowly than light. Thus there is no true maximum subluminal energy if we consider both spin states. Nonetheless, because the helicity is not a constant of motion, we can extract bounds from the IC data.

It is natural to expect that in an astrophysical source, energy will be roughly evenly distributed between left- and right-handed electrons. Yet we may worry that because of the differing kinematics of the two helicities, the energy may not be so evenly distributed. However, even if the initial distribution of electrons were completely polarized at the 
highest energies (with only one helicity state occupied), the presence of a magnetic field in a source will destroy this polarization. Because an electron has an anomalous magnetic moment, the spin will precess during cyclotron motion, changing by $\mathcal{O}\left(e^{2} \gamma\right)$ with each revolution. Moreover, since the MAV is direction- as well as spin-dependent, a particle with fixed helicity and energy may be subluminal when moving in certain directions along its trajectory and superluminal when moving in other directions. So if we can infer the presence of electrons of a given energy in a source, there must be electrons of that energy with both helicities. Further, if there is no evidence of vacuum Cerenkov radiation, then both helicities at that energy must be moving more slowly than light.

If we observe IC photons with energies up to $E_{\max }$ coming at us from a source along the direction $\hat{e}$, there must be electrons equally energetic in the source, also moving in the $\hat{e}$ direction. If the radiation from the source is well understood, and vacuum Cerenkov radiation is not present, then it follows that

$$
\left|d_{0 j} \hat{e}_{j}+d_{j k} \hat{e}_{j} \hat{e}_{k}\right|<\frac{1}{2\left(E_{\max } / m\right)^{2}} .
$$

If we were to include the effects of $c$, we would find

$$
-c_{0 j} \hat{e}_{j}-c_{j k} \hat{e}_{j} \hat{e}_{k}+\left|d_{0 j} \hat{e}_{j}+d_{j k} \hat{e}_{j} \hat{e}_{k}\right|<\frac{1}{2\left(E_{\max } / m\right)^{2}}
$$

(It immediately follows that including the effects of $d$ would only strengthen the previously derived IC bounds on $c$.) However, there are good reasons to believe that the magnitude of $c$ is at least as small as the bounds we shall be placing on $d$ here. The synchrotron spectrum is less sensitive to $d$ than to $c$, and although the synchrotron data only give one-sided bounds on $c$, they are suggestive the overall order of magnitude that $c$ may be. Even stronger restrictions on $c$ come from naturalness conditions, since there are much stronger bounds on $c$-at the $10^{-25}$ level-for the proton [23]. Radiative corrections mix the $c$ coefficients for different charged particles, so it would be highly unnatural for the electron $c$ to be many orders of magnitude larger than the proton $c$. We shall therefore assume than $c$ can be neglected compared to $d$ in what follows.

Direct experimental bounds on the $d$ coefficients in the electron and proton sectors are not nearly as good. In our formulation with $d_{\nu T}=0$, there are bounds only on $d_{T X}$ and $d_{T Y}$, and these are mixed with bounds on $H$. However, the bounds in the electron sector are at the $10^{-19}$ level [5], which is substantially better than we can achieve with the $\mathrm{IC}$ data. Assuming that there is no special cancellation between $d$ and $H$, a reasonably conservative interpretation of these bounds is that $\left|d_{T X}\right|$ and $\left|d_{T Y}\right|$ must be less than $10^{-18}$. (In earlier formulations where the fermions were not quantized in the sun-centered frame, and in which $d_{\nu T}$ was therefore nonvanishing, bounds on $d_{T \nu}$ became entangled with bounds on $d_{\nu T}$ and $b_{\nu}$. Here, we have avoided this complication by choosing the formulation of the theory that has the most concise expression when the effects of $d$ are predominant.) 


\begin{tabular}{|l|c|c|c|c|}
\hline Emission source & $\hat{e}_{X}$ & $\hat{e}_{Y}$ & $\hat{e}_{Z}$ & $E_{\max } / m$ \\
\hline Crab nebula & -0.10 & -0.92 & -0.37 & $2 \times 10^{8}[24]$ \\
G 0.9+0.1 & 0.05 & 0.88 & 0.47 & $10^{7}[25]$ \\
G 12.82-0.02 & -0.06 & 0.95 & 0.29 & $5 \times 10^{7}[26]$ \\
G 18.0-0.7 & -0.11 & 0.97 & 0.24 & $7 \times 10^{7}[27,[28]$ \\
G 347.3-0.5 & 0.16 & 0.75 & 0.64 & $2 \times 10^{7 *}[29]$ \\
MSH 15-52 & 0.34 & 0.38 & 0.86 & $8 \times 10^{7}[30]$ \\
Mkn 421 & 0.76 & -0.19 & -0.62 & $3 \times 10^{7 *}[31,[32]$ \\
Mkn 501 & 0.22 & 0.74 & -0.64 & $4 \times 10^{7 *}[33]$ \\
PSR B1259-63 & 0.42 & 0.12 & 0.90 & $6 \times 10^{6 *}[34]$ \\
SNR 1006 AD & 0.52 & 0.53 & 0.67 & $7 \times 10^{6}[35]$ \\
Vela SNR & 0.44 & -0.55 & 0.71 & $1.3 \times 10^{8}[36]$ \\
\hline
\end{tabular}

Table 1: Parameters for the IC sources that we shall use to constrain $d$. References are given for each value of $E_{\max }$. The four $E_{\max } / m$ values marked with asterisks denote the four sources for which the IC origin of the observed $\gamma$-rays is merely strongly favored, rather than completely assured.

Bounds on an electron MAV less than one have also been derived from looking at the threshold for the pair creation process $\gamma \rightarrow e^{+}+e^{-}$, which is forbidden if Lorentz symmetry is exact. This can provide excellent bounds on a $c$ term, but not on a $d$. The bounds on $c$ come from observations of $\mathrm{TeV}$ photons from sources such as the Crab nebula. If the MAV for both electron spin states is less than one, then a very energetic photon may decay into two electrons, with a decay rate of $\mathcal{O}\left(e^{2}\right)$. If we observe photons from distant sources up to a given energy, we know that this decay is not possible up to at least the measured energy. However, the situation is not the same with $d$, because of angular momentum complications. All the particles in the process $\gamma \rightarrow e^{+}+e^{-}$are nearly collinear, so if angular momentum is conserved (which it nearly is), the helicities of all three particles must be the same - a right-handed photon decays to two right-handed electrons, and the same with left-handed particles. However, because the electron MAV with $d$ depends on the helicity, the process will be kinematically allowed only for one of the two helicity options outlined above. If the process is allowed for right-handed quanta, it is not allowed for left-handed ones, and so some half of the photons coming from a given source will not decay into electron-positron pairs. (Although angular-momentum is not exactly conserved in Lorentz-violating theories, it is still approximately conserved. Decays that do not conserve angular momentum will be suppressed by two factors of the Lorentz-violating coefficient $d$, since $d$ would need to appear explicitly in the amplitude for the process. Because $d$ is expected to be miniscule, such decays should be quite slow to happen.)

So an analysis of IC data appears to offer the best prospects for bounding $d$. We have 


\begin{tabular}{|c|c|c|}
\hline$\left|d_{\nu \mu}\right|$ & Bound (no priors) & Bound (with priors) \\
\hline$\left|d_{X X}\right|$ & $8 \times 10^{-14}$ & $2 \times 10^{-14}$ \\
$\left|d_{Y Y}\right|$ & $7 \times 10^{-15}$ & $3 \times 10^{-15}$ \\
$\left|d_{Z Z}\right|$ & $2 \times 10^{-14}$ & $3 \times 10^{-15}$ \\
$\left|d_{(X Y)}\right|$ & $5 \times 10^{-14}$ & $2 \times 10^{-15}$ \\
$\left|d_{(Y Z)}\right|$ & $7 \times 10^{-14}$ & $2 \times 10^{-14}$ \\
$\left|d_{(Y Z)}\right|$ & $2 \times 10^{-14}$ & $7 \times 10^{-15}$ \\
$\left|d_{T X}\right|$ & $5 \times 10^{-14}$ & - \\
$\left|d_{T Y}\right|$ & $5 \times 10^{-15}$ & - \\
$\left|d_{T Z}\right|$ & $4 \times 10^{-16}$ & $8 \times 10^{-17}$ \\
\hline
\end{tabular}

Table 2: Independent bounds on the components of $d$, as determined both with and without the inclusion of the earlier bounds on $\left|d_{T X}\right|$ and $\left|d_{T Y}\right|$ coming from clock comparison experiments [5].

previously collected a number of data points from the observational literature. These are replicated in table 1. The data come from sources of TeV IC photons. (In a few cases, it is not completely certain - although it does appear strongly favored - that the high-energy spectrum in entirely due to IC emission.) $E_{\max }$ is the highest observed photon energy from each of these sources. These sources all have well-understood spectra, with no indications of the vacuum Cerenkov radiation we would expect to see if there were superluminal electrons. Therefore, each $E_{\max }$ is a lower bound on the maximum subluminal energy for electrons moving in the source-to-Earth direction $\hat{e}$. The components of each $\hat{e}$ are given in the sun-centered coordinate system.

The data from table 1, when combined with inequality (10), give eleven two-sided bounds on various combinations of the $d$ coefficients. This is enough to bound each of the six $d_{(j k)}=d_{j k}+d_{k j}$ and the three $d_{T j}$ coefficients above and below. The bounds of the form (10) may be translated into bounds on the separate coefficients by means of linear programming. The results are shown in table 2. Unlike with previous results for $c$, where synchrotron and IC data points each provided half of a two-sided inequality, both the upper and lower limits on each combination of $d$ coefficients come from a single data point. Because of this, the separate bounds on the various $d_{\nu \mu}$ are all symmetric about zero.

The first column of numbers in table 2 contains the bounds coming solely from the IC data. These are the absolute maximum values that each $\left|d_{\nu \mu}\right|$ may take when the components are subject to all eleven of the inequalities (10). The second set of bounds were calculated using the same method, but this time the linear program also incorporated priors from the clock comparison data. The clock comparison bounds on $d_{T X}$ and $d_{T Y}$ are several orders of magnitude better than the astrophysical bounds, so the second set of bounds are effectively just the limits on the various $\left|d_{\nu \mu}\right|$ when $d_{T X}$ and $d_{T Y}$ are set 
to zero. Including these priors improves the bounds on the other coefficients by factors of two to twenty-five. The bounds on $d$, with the clock comparison priors, are roughly comparable to the best bounds on $c$ (which are mostly astrophysical, but also incorporate some different laboratory results). This is not surprising, since the tightness of each set of bounds is determined by the highest electron energies in the sources we can observe. If it turns out that the magnitude of $c$ is actually right at the $10^{-15}$ level implied by the best bounds, then including the effects of $c$ according to (11) would worsen the bounds on $d$ slightly, but by no more than an $\mathcal{O}(1)$ factor.

The electron $c$ coefficients have been bounded using analyses of synchrotron spectra, analyses of IC spectra, and the observed absence of the process $\gamma \rightarrow e^{+}+e^{-}$. The scales of these bounds are all roughly similar, as they are all ultimately determined by the same basic quantity, which is the maximum energy attained by individual particles in energetic sources. However, the $d$ terms have been harder to constrain. Of the astrophysical data mentioned above, only the IC data really provide good bounds on $d$. Our new IC bounds on $d$ include bounds on seven Lorentz-violating coefficients that have not previously been bounded, at a $10^{-15}$ level comparable to the best astrophysical bounds on $c$.

\section{Acknowledgments}

The author is grateful to V. A. Kostelecký for helpful discussions. This work is supported in part by funds provided by the U. S. Department of Energy (D.O.E.) under cooperative research agreement DE-FG02-91ER40661.

\section{References}

[1] D. Colladay, V. A. Kostelecký, Phys. Rev. D 55, 6760 (1997).

[2] D. Colladay, V. A. Kostelecký, Phys. Rev. D 58, 116002 (1998).

[3] V. A. Kostelecký, Phys. Rev. D 69, 105009 (2004).

[4] C. J. Berglund, L. R. Hunter, D. Krause, Jr., E. O. Prigge, M. S. Ronfeldt, S. K. Lamoreaux, Phys. Rev. Lett. 75, 1879 (1995).

[5] V. A. Kostelecký, C. D. Lane, Phys. Rev. D 60, 116010 (1999).

[6] L.-S. Hou, W.-T. Ni, Y.-C. M. Li, Phys. Rev. Lett. 90, 201101 (2003).

[7] B. R. Heckel, C. E. Cramer, T. S. Cook, S. Schlamminger, E. G. Adelberger, U. Schmidt, Phys. Rev. Lett. 97, 021603 (2006).

[8] P. Antonini, M. Okhapkin, E. Göklü, S. Schiller, Phys. Rev. A 71, 050101 (2005). 
[9] P. L. Stanwix, M. E. Tobar, P. Wolf, M. Susli, C. R. Locke, E. N. Ivanov, J. Winterflood, F. van Kann, Phys. Rev. Lett. 95, 040404 (2005).

[10] S. Herrmann, A. Senger, E. Kovalchuk, H. Müller, A. Peters, Phys. Rev. Lett. 95, 150401 (2005).

[11] H. Müller, Phys. Rev. D 71, 045004 (2005).

[12] G. Saathoff, S. Karpuk, U. Eisenbarth, G. Huber, S. Krohn, R. Muñoz Horta, S. Reinhardt, D. Schwalm, A. Wolf, G. Gwinner, Phys. Rev. Lett. 91, 190403 (2003).

[13] C. D. Lane, Phys. Rev. D 72, 016005 (2005).

[14] F. W. Stecker, S. L. Glashow, Astropart. Phys. 16, 97 (2001).

[15] T. Jacobson, S. Liberati, D. Mattingly, Nature 424, 1019 (2003).

[16] T. Jacobson, S. Liberati, D. Mattingly, F. W. Stecker, Phys. Rev. Lett. 93, 021101 (2004).

[17] B. Altschul, Phys. Rev. Lett. 96, 201101 (2006).

[18] B. Altschul, Phys. Rev. D 74, 083003 (2006).

[19] B. Altschul, J. Phys. A 39, 13757 (2006).

[20] R. Bluhm, V. A. Kostelecký, C. D. Lane, N. Russell, Phys. Rev. D 68, 125008 (2003).

[21] V. A. Kostelecký, R. Lehnert, Phys. Rev. D 63, 065008 (2001).

[22] B. Altschul, D. Colladay, Phys. Rev. D 71, 125015 (2005).

[23] P. Wolf, F. Chapelet, S. Bize, A. Clairon, Phys. Rev. Lett. 96, 060801 (2006).

[24] T. Tanimori, et al., 1998, Astrophys. J. 492, L33 (1998).

[25] F. A. Aharonian, et al., Astron. Astrophys. 432, L25 (2005).

[26] F. A. Aharonian, et al., Astrophys. J. 636, 777 (2006).

[27] F. A. Aharonian, et al., Astron. Astrophys. 442, L25 (2005).

[28] F. A. Aharonian, et al., astro-ph/0607548.

[29] F. A. Aharonian, et al., Nature 432, 75 (2004).

[30] F. A. Aharonian, et al., Astron. Astrophys. 435, L17 (2005). 
[31] J. Albert, et al., astro-ph/0603478.

[32] F. A. Aharonian, et al., Astron. Astrophys. 437, 95 (2005).

[33] F. A. Aharonian, et al., Astron. Astrophys. 349, 11 (1999).

[34] F. A. Aharonian, et al., Astron. Astrophys. 442, 1 (2005).

[35] G. E. Allen, R. Petre, E. V. Gotthelf, Astrophys. J. 558, 739 (2001).

[36] F. A. Aharonian, et al., Astron. Astrophys. 448, L43 (2006). 